\title{
Ground State Energy of Massive Scalar Field in the Background of Finite Thickness Cosmic String
}

\author{
N.R. Khusnutdinov ${ }^{1) *}$ AND M. BordaG ${ }^{2) \dagger}$ \\ 1) Department of Physics, Kazan State Pedagogical University, \\ Mezhlauk 1, 420021, Kazan, Russia, and \\ Engelhardt Observatory of Kazan, 422526, EAO, Russia \\ 2) Universität Leipzig, Institut für Theoretische Physik \\ Augustusplatz 10/11, 04109 Leipzig, Germany
}

August 12, 2018

\begin{abstract}
We calculate the ground state energy of a massive scalar field in the background of a cosmic string of finite thickness (Gott-Hiscock metric). Using zeta functional regularization we discuss the renormalization and the relevant heat kernel coefficients in detail. The finite (non local) part of the ground state energy is calculated in $2+1$ dimensions in the approximation of a small mass density of the string. By a numerical calculation it is shown to vanish as a function of the radius of the string and of the parameter $\xi$ of the nonconformal coupling.
\end{abstract}

\section{Introduction}

The Universe may have undergone a number of phase transitions since the big bang due to the spontaneous symmetry breaking in gauge theories. A number of topological objects may have been produced during the expansion of the Universe [1] amongst which the cosmic strings seem to be of particular interest as seeds for the density fluctuations in the early Universe that are responsible for the

*e-mail:nail@dtp.ksu.ras.ru

${ }^{\dagger}$ e-mail: Michael.Bordag@itp.uni-leipzig.de 
formation of galaxies (see for example [2]). Also, the gravitational radiation produced by the formation of cosmic strings is part of cosmological scenarios.

Several models of strings have been suggested. First of all Vilenkin has investigated the case of an infinitely thin cosmic string [3]. The energy momentum tensor of this string has a delta function like singular form. The space-time is locally flat except in the origin where it has a delta-shaped Riemann tensor [4]. The vacuum expectation value of the stress-energy tensor for different kinds of fields in that background has been calculated both for zero temperature [5] and non-zero temperature [6] cases.

The vanishing thickness of the string causes known problems. The vacuum expectation value of the stress - energy tensor has a non - integrabile singularity in the origin which can be seen already from dimensional considerations. As a consequence, for the renormalization of the ground state energy of quantum fields an additional counterterm is required. It is known as the topological Kac term [7]. This additional part may be recognized as due to boundary condition at the origin [6].

The mentioned problems can be avoided by considering a string with finite thickness. The simplest case is that of a constant matter density inside the string. It has been considered in Refs. [8, 9]. The pressure $p$ and energy density $\mathcal{E}$ inside the string obey the condition $p+\mathcal{E}=0$. The exterior of this string is a conical space-time and the interior is a constant curvature space ("cup" space). The metric is smoothly matched on the surface of the string but the scalar curvature has a laps on it. In fact, this space is a cone with a smoothed origin. There is no gravitational field outside the string in both above cases in opposite to the Newtonian logarithmic gravitational potential of a thread-like matter distribution. Note that this statement remains valid for an arbitrary radial matter distribution inside the string, provided that the translational invariance along the string is not broken.

The purpose of this paper is to calculate the ground state energy of a massive scalar field in the background of a finite thickness cosmic string.

In fact, we consider the $(2+1)$ dimensional case. In zetafunctional regularization, the ground state energy of a scalar field $\Phi$ is given by

$$
E_{0}=M^{2 s} \frac{1}{2} \zeta\left(s-\frac{1}{2}\right),
$$

where

$$
\zeta(s)=\sum_{(n)}\left(\lambda_{(n)}+m^{2}\right)^{-s}
$$

is the zetafunction of the corresponding Laplace operator. The parameter $M$ is arbitrary. It has the dimension of a mass. Usually it is denoted by $\mu$ which we reserve here for the linear mass density of the string. We assume the field $\Phi$ to be put into a large volume with Dirichlet boundary conditions in order to render the eigenvalues discrete. It will be seen that the influence of this boundary 
separates completely from the contributions of the pure background. The $\lambda_{(n)}$ are eigenvalues of the two dimensional Laplace operator

$$
(\Delta-\xi \mathrm{R}) \varphi_{(n)}(x)=\lambda_{(n)} \varphi_{(n)}(x) .
$$

where $\mathrm{R}$ is the curvature scalar.

In the $(3+1)$ dimensional case we would have to add the integration over the momentum of the translational invariant direction along the axis of the string and get

$$
E_{0}^{(3+1)}=\frac{\sqrt{\pi}}{2} \frac{\Gamma(s-1)}{\Gamma(s-1 / 2)} M^{2 s} \zeta(s-1) .
$$

The ultraviolet divergencies of the ground state energy are completely determined by the first few heat kernel coefficients. By means of

$$
\zeta(s)=\int_{0}^{\infty} \frac{\mathrm{d} t}{t} \frac{t^{s}}{\Gamma(s)} K(t)
$$

and the asymptotic expansion for $t \rightarrow 0$

$$
K(t)=\frac{1}{4 \pi t} \sum_{n \geq 0} B_{n} t^{n}
$$

of the heat kernel $K(t)$ corresponding to the operator in Eq. (3) the divergent part of the ground state energy can be expressed in terms of the first four coefficients (in $(3+1)$ dimensions five coefficients would enter). We define

$$
\begin{aligned}
E_{0}^{\operatorname{div}}(s)= & \left(\frac{M}{m}\right)^{2 s} \frac{1}{8 \pi}\left\{\frac{\Gamma(s-3 / 2)}{\Gamma(s-1 / 2)} B_{0} m^{3}+B_{1 / 2} \frac{\Gamma(s-1) m^{2}}{\Gamma(s-1 / 2)}+B_{1} m\right. \\
& \left.+B_{3 / 2} \frac{\Gamma(s)}{\Gamma(s-1 / 2)}\right\} .
\end{aligned}
$$

In the following we calculate these coefficients for a string of zero thickness, reobtaining known results, and for a string of finite thickness in the approximation of a small mass density of the string. Using these coefficients, the renormalization of the ground state energy can be carried out in the standard way by adding the counterterms corresponding to (7) to a suitably defined classical energy. So we get the renormalized ground state energy

$$
E_{0}^{\mathrm{ren}}=\lim _{s \rightarrow 0}\left(E_{0}-E_{0}^{\mathrm{div}}\right)
$$

In the $(2+1)$ dimensional case we obtain the result $E_{0}^{\text {ren }}=0$ in the given order of small mass density numerically.

We note that the ground state energy defined in this way obeys the normalization condition

$$
E_{0}^{\text {ren }} \rightarrow 0 \text { for } m \rightarrow \infty
$$


which follows, from the circumstance that the heat kernel expansion is at once the asymptotic expansion for large mass.

The organization of the papers as follows. In Sec.2 we describe the GottHiscock space-time of finite thickness cosmic string. In Sec.3 we write down the general formulas for the thin string and calculate the corresponding heat kernel coefficients. In the next section we do the same for the finite thickness string. In Sec.5 we calculate the ground state energy in the approximation of a small angle deficit. The result is discussed in Sec.6 and an appendix contains some technical details of numerical calculations.

We use units $\hbar=c=G=1$.

\section{The Space-Time}

The metric of Gott - Hiscock [8, 9] is a solution of Einstein's equations and it describes the space-time of an infinitely long straight cosmic string with non vanishing thickness. The energy density $\mathcal{E}$ is constant inside the string and it is zero outside of it. The manifold can be covered by two maps. The first map, $t \in$ $(-\infty,+\infty), \rho \in\left[0, \rho_{0}\right], \varphi \in[0,2 \pi], z \in(-\infty,+\infty)$, covers the interior of the string and the second one, $t \in(-\infty,+\infty), r \in\left[r_{0},+\infty\right), \varphi \in[0,2 \pi], z \in(-\infty,+\infty)$, covers the exterior. The coordinates $(t, \varphi, z)$ are the same in both maps. $r_{0}$ and $\rho_{0}$ denote the radius of the string in external and internal coordinates, respectively. The string is situated along the $z$-axis. The metrics are $C^{1}$ - matched on the surface of the string, there is no surface stress energy (the extrinsic curvature tensors of the interior and exterior metrics are equal to each other [9]). The metric has the following form

$$
d s_{i n}^{2}=d t^{2}-d \rho^{2}-\rho_{*}^{2} \sin ^{2}\left(\frac{\rho}{\rho_{*}}\right) d \varphi^{2}-d z^{2},
$$

inside the string, and

$$
d s_{\text {out }}^{2}=d t^{2}-d r^{2}-\frac{r^{2}}{\nu^{2}} d \varphi^{2}-d z^{2}
$$

outside of it. Here $\rho_{*}=1 / \sqrt{8 \pi \mathcal{E}}$ is 'energetic' radius of the string; $\mathcal{E}$ is the energy density inside the string. The matching condition on the surface of the string links the exterior parameters $\left(\nu, r_{0}\right)$ and interior ones $\left(\rho_{*}, \rho_{0}\right)$ of the string

$$
\frac{\rho_{0}}{\rho_{*}} \stackrel{\text { def }}{=} \epsilon=\text { const }, \nu=\frac{1}{\cos \epsilon}, \frac{r_{0}}{\rho_{0}}=\frac{\tan \epsilon}{\epsilon} .
$$

From these relations we have the following consequences. The limit to the Minkowski space-time is achieved by letting the energy density inside the string tend to zero $\left(\rho_{*} \rightarrow \infty\right)$ for fixed radius of the string $\rho_{0}$. Then the angle deficit will tend to zero too because of $\epsilon \rightarrow 0$. Thereby in this limit $\nu=1 / \cos \epsilon=1, r_{0}=\rho_{0}$ 
and both metrics turn into Minkowski space time. On the other hand side, in order to shrink the string $\left(\rho_{0} \rightarrow 0\right)$ at fixed exterior $(\epsilon$ resp. the angle deficite are constant) we must turn the energy density $\mathcal{E}$ to infinity proportional to $\epsilon^{2} / 8 \pi \rho_{0}^{2}$. Nevertheless the energy $\mu$ per unit length of the string $\mu$ which is the product of the energy density $\epsilon^{2} / 8 \pi \rho_{0}^{2}$ and the cross section of the string is always constant and equals $(1-1 / \nu) / 4$, the same value as for the infinitely thin cosmic string and it doesn't depend on the radius of the string. The two dimensional part $(t=$ const, $z=$ const $)$ of the space-time (10, 11) is depicted in Fig.1.

The manifold can be covered also by one map. One can continue the exterior radial coordinate $r$ into the interior of the string by mapping $r=r_{0}+$ $\left(\rho_{0} / \epsilon\right) \tan \left(\epsilon \rho / \rho_{0}-\epsilon\right)$ and the space-time will be described by the metric

$$
\begin{aligned}
d s_{\text {in }}^{2} & =d t^{2}-\frac{d r^{2}}{\left[1+\epsilon^{2}\left(r-r_{0}\right)^{2} / \rho_{0}^{2}\right]^{2}}-\frac{r^{2}}{\nu^{2}} \frac{d \varphi^{2}}{1+\epsilon^{2}\left(r-r_{0}\right)^{2} / \rho_{0}^{2}}-d z^{2}, r \in\left[0, r_{0}\right], \\
d s_{\text {out }}^{2} & =d t^{2}-d r^{2}-\frac{r^{2}}{\nu^{2}} d \varphi^{2}-d z^{2}, r \in\left[r_{0}, \infty\right) .
\end{aligned}
$$

Here, the parameters $\nu, \rho_{0}$ and $r_{0}$ are connected by condition (12). Nevertheless we shall use the metric in two maps because it is simpler for calculations. As far as the angle deficit is fixed, that is $\epsilon=\arccos 1 / \nu=\rho_{0} / \rho_{*}$ is constant, one can exclude $\rho_{*}$ and rewrite the metric in the form which will be used in the following

$$
d s_{i n}^{2}=d t^{2}-d \rho^{2}-\frac{\rho_{0}^{2}}{\epsilon^{2}} \sin ^{2}\left(\frac{\epsilon \rho}{\rho_{0}}\right) d \varphi^{2}-d z^{2}, \rho \in\left[0, \rho_{0}\right]
$$

inside the string, and

$$
d s_{\text {out }}^{2}=d t^{2}-d r^{2}-\frac{r^{2}}{\nu^{2}} d \varphi^{2}-d z^{2}, r \in\left[r_{0}, \infty\right)
$$

outside of it.

\section{Infinitely thin cosmic string}

The metric for an infinitely thin cosmic string is given by (14) for $r \in[0, \infty)$. In the $(2+1)$ dimensional case, which we consider here, the coordinate $z$ is absent. The eigenvalues $\lambda_{(n)}$ which enter the zeta function (2) are determined by Eq. (3) in the background of the spatial part of this metric. The curvature scalar $\mathrm{R}$ in this equation is proportional to the two dimensional delta function. So it is a potential with pointlike support and should be taken into account by a self adjoint extension of the operator corresponding to Eq. (3). Instead, we drop these contributions here by considering the case $\xi=0$ only.

By means of the ansatz

$$
\varphi_{(n)}=e^{i n \varphi} \sqrt{\frac{\nu}{r}} \mathcal{R}(r),
$$




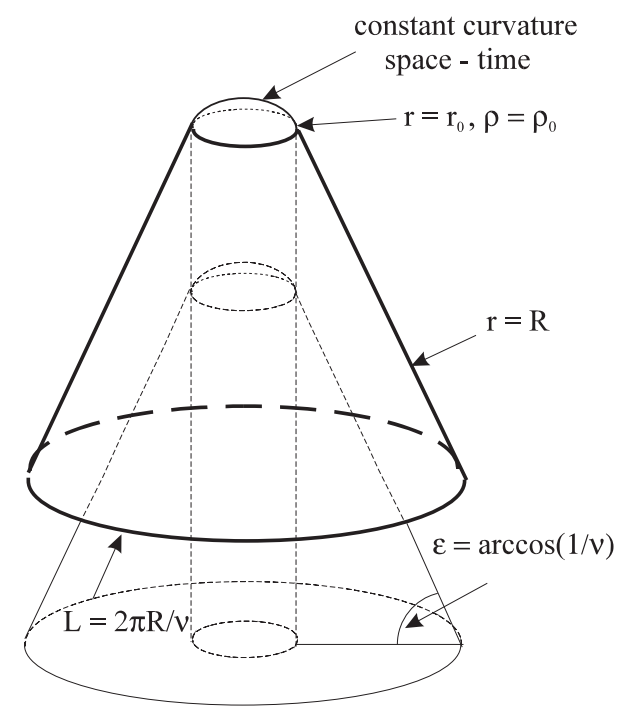

Figure 1: Plot of two-dimensional ( $t=$ const, $z=$ const) part of finite thickness cosmic string space-time

$(n=0, \pm 1, \pm 2, \ldots)$ we arrive at the radial equation

$$
\left(\frac{d^{2}}{d r^{2}}-\frac{n^{2} \nu^{2}-1 / 4}{r^{2}}+\lambda^{2}\right) \mathcal{R}=0
$$

The solution regular at the origin of this equation is a Bessel function

$$
\mathcal{R}=\sqrt{\frac{\pi \lambda r}{2}} J_{n \nu}(\lambda r)
$$

We assume it to obey Dirichlet boundary conditions at $r=R$. Then the solutions $\lambda=\lambda_{n, i}(i=1,2, \ldots)$ of the equation

$$
\sqrt{\frac{\pi \lambda R}{2}} J_{n \nu}(\lambda R)=0
$$

are the discrete eigenvalues $\lambda \rightarrow \lambda_{(n)}=\lambda_{n, i}$. Now the ground state energy and equally the zeta function (2) can be written in the form

$$
E_{0}^{\text {thin }}(s)=\frac{1}{2} M^{2 s} \sum_{i=1}^{\infty} \sum_{n=-\infty}^{\infty}\left(\lambda_{n, i}+m^{2}\right)^{1 / 2-s}
$$

The sum over $i$ can be rewritten as an integral (for details see [10])

$$
E_{0}^{\text {thin }}(s)=-M^{2 s} \frac{\cos (\pi s)}{2 \pi} \sum_{n=0}^{\infty} d_{n} \int_{m}^{\infty} d k\left(k^{2}-m^{2}\right)^{1 / 2-s} \frac{\partial}{\partial k} \ln k^{-n \nu} I_{n \nu}(k R),
$$


where $d_{n>0}=2$ and $d_{0}=1$ is the multiplicity of the angular momentum. Note the factor $k^{-n \nu}$ in the argument of the logarithm, see [10.

In order to investigate the pole of $E_{0}^{\text {thin }}(s)$ we use the uniform asymptotic expansion of the modified Bessel function for $n \rightarrow \infty$ [11]

$$
I_{n \nu}(n \nu z)=\sqrt{\frac{t}{2 \pi n \nu}} e^{n \nu \eta(z)}\left\{1+\sum_{k=1}^{\infty} \frac{u_{k}(t)}{(n \nu)^{k}}\right\}
$$

with $t=1 / \sqrt{1+z^{2}}, \eta(z)=\sqrt{1+z^{2}}+\ln \left(z /\left(1+\sqrt{1+z^{2}}\right)\right)$ and $z=k R / n \nu$.

The pole term of the zeta function $\zeta(s)$ (2) in $s=-1 / 2$ resp. of the energy $E_{0}(s)$ in $s=0$ will be delivered by the first few terms (up to $k=2$ ) of this expansion when inserting them into $E_{0}(20)$. We note that this expansion is at once an asymptotic expansion for large masses and that the individual heat kernel coefficients enter expansions like (7) multiplied by the corresponding power of $m$. Therefore we will in the following keep track of these powers and drop all contributions which for $m \rightarrow \infty$ are of order $O\left(\frac{1}{m}\right)$.

After inserting the expansion (21) into (20), the integration over $k$ can be carried out using

$$
\int_{1}^{\infty} d x\left(x^{2}-1\right)^{1 / 2-s} x\left(1+x^{2} / \alpha^{2}\right)^{-p / 2}=\frac{\Gamma\left(\frac{3}{2}-s\right) \Gamma\left(s+\frac{p-3}{2}\right)}{2 \Gamma\left(\frac{p}{2}\right)} \alpha^{p}\left(1+\alpha^{2}\right)^{-s-\frac{p-3}{2}},
$$

and we obtain the following expression for $E(s)$

$$
\begin{aligned}
E(s)= & -\frac{\cos \pi s}{4 \pi}\left(\frac{M}{m}\right)^{2 s} R m^{2} \Gamma\left(\frac{3}{2}-s\right)\left\{\sum_{n=0}^{\infty} d_{n}\left[\frac{\Gamma(s-1)}{\sqrt{\pi}}{ }_{2} F_{1}-\frac{n \nu}{b} \Gamma\left(s-\frac{1}{2}\right)\right]\right. \\
& -\frac{\Gamma\left(s-\frac{1}{2}\right)}{2 b} Z\left(0, s-\frac{1}{2}\right)-\frac{1}{4 b^{2} \sqrt{\pi}}\left[\Gamma(s) Z(0, s)-\frac{10}{3} \frac{\Gamma(s+1)}{b^{2}} Z(2, s+1)\right] \\
& -\frac{1}{8 b^{3}}\left[\Gamma\left(s+\frac{1}{2}\right) Z\left(0, s+\frac{1}{2}\right)-\frac{6}{b^{2}} \Gamma\left(s+\frac{3}{2}\right) Z\left(2, s+\frac{3}{2}\right)\right. \\
& \left.+\frac{5}{2 b^{4}} \Gamma\left(s+\frac{5}{2}\right) Z\left(4, s+\frac{5}{2}\right)\right]-\frac{1}{96 b^{4} \sqrt{\pi}}[25 \Gamma(s+1) Z(0, s+1) \\
& -\frac{1062}{5 b^{2}} \Gamma(s+2) Z(2, s+2)+\frac{884}{5 b^{4}} \Gamma(s+3) Z(4, s+3) \\
& \left.\left.-\frac{1768}{63 b^{6}} \Gamma(s+4) Z(6, s+4)\right]+\ldots\right\} .
\end{aligned}
$$

Here ${ }_{2} F_{1}={ }_{2} F_{1}\left(-\frac{1}{2}, s-1 ; \frac{1}{2} ;-\left(\frac{n \nu}{b}\right)^{2}\right)$ is the hypergeometric function; $Z(p, q)=$ $\sum_{n=0}^{\infty} d_{n}(n \nu)^{p}\left(1+\left(\frac{n \nu}{b}\right)^{2}\right)^{-q}$ and $b=m R$. Next we have to perform the analytical continuation $s \rightarrow 0$.

First of all let's consider the part containing the hypergeometric function

$$
Y(s)=\sum_{n=0}^{\infty} d_{n}\left[\frac{\Gamma(s-1)}{\sqrt{\pi}}{ }_{2} F_{1}-\frac{n \nu}{b} \Gamma\left(s-\frac{1}{2}\right)\right]
$$




$$
=\frac{\Gamma(s-1)}{\sqrt{\pi}}+2 \sum_{n=1}^{\infty}\left[\frac{\Gamma(s-1)}{\sqrt{\pi}}{ }_{2} F_{1}-\frac{n \nu}{b} \Gamma\left(s-\frac{1}{2}\right)\right] .
$$

For the calculation of the series we use the Mellin - Barnes type representation of the hypergeometric function

$$
\begin{aligned}
& \frac{\Gamma(s-1)}{\sqrt{\pi}}{ }_{2} F_{1}\left(-\frac{1}{2}, s-1 ; \frac{1}{2} ;-\left(\frac{n \nu}{b}\right)^{2}\right) \\
= & \frac{1}{\Gamma\left(-\frac{1}{2}\right)} \frac{1}{2 \pi i} \int_{\gamma} \frac{\Gamma(s-1+t)}{t-1 / 2} \Gamma(-t)\left(\frac{n \nu}{b}\right)^{2 t} d t,
\end{aligned}
$$

where the contour is such that the poles of $\Gamma(s-1+t) /(t-1 / 2)$ lie to the left of it and the poles of $\Gamma(-t)$ to the right [11]. Before interchanging sum and integral one has to shift the contour $\gamma$ to the left crossing the pole at $t=1 / 2$ up to $t=-1 / 2$ and then to close it to the left. This is because of the convergence of the series $\sum_{n=1}^{\infty} n^{2 t}$ it is necessary to have $t<-1 / 2$. The residue at the point $t=1 / 2$ cancels the second manifestly divergent term in the sum (23). Taking the limit $s \rightarrow 0$ we obtain the following finite expression for (23)

$$
\begin{aligned}
Y(0)= & \frac{4}{\sqrt{\pi}}\left(\frac{\nu}{b}\right)^{2} \zeta_{R}^{\prime}(-2)+\frac{1}{\sqrt{\pi}}\left(2 \ln \frac{2 \pi b}{\nu}-3\right) \\
& +\frac{1}{\sqrt{\pi}} \sum_{n=0}^{\infty} \frac{(-1)^{n}}{(n+1)(n+2)} \frac{\zeta_{R}(2 n+2)}{n+3 / 2}\left(\frac{b}{\nu}\right)^{2+2 n} .
\end{aligned}
$$

Here $\zeta_{R}(a)$ is Riemann zeta function.

The series is absolutely convergent for $b / \nu<1$, but we need it in the domain of large $R$, that is for $b \gg 1$. For this reason we perform the analytic continuation into the domain we need. With $t=i b / \nu$ in the formula

$$
\sum_{n=1}^{\infty} \frac{t^{2 n}}{n} \zeta_{R}(2 n)=\ln \frac{\pi t}{\sin \pi t},|t|<1,
$$

we can express the series in $Y(0)$ in terms of the series (24). As a result, $Y(0)$ is expressed in terms of functions which are analytical in the whole plane of $b$ and it can be divided into a polynomial and an exponentially small part

$$
Y(0)=\frac{2 \sqrt{\pi}}{3} \frac{b}{\nu}-\frac{\sqrt{\pi} \nu}{3 b}+\frac{2}{\sqrt{\pi}} \ln \left(1-e^{-\frac{2 \pi b}{\nu}}\right)-2 \sqrt{\pi}\left[4 \widetilde{Q}_{1}(b / \nu)-2 \widetilde{Q}_{2}(b / \nu)\right]
$$

with

$$
\widetilde{Q}_{a}(x)=\frac{1}{x^{a}} \int_{0}^{x} \frac{d t t^{a}}{e^{2 \pi t}-1}-\frac{1}{x^{a}} \frac{\Gamma(a+1)}{(2 \pi)^{a+1}} \zeta_{R}(a+1)=-\frac{1}{x^{a}} \int_{x}^{\infty} \frac{d t t^{a}}{e^{2 \pi t}-1} .
$$

For large $b$ this expression is exponentially small. Therefor we arrive at

$$
Y(0)=\frac{2 \sqrt{\pi}}{3} \frac{b}{\nu}-\frac{\sqrt{\pi} \nu}{3 b}+O\left(e^{-b}\right)
$$


The same result may be obtained in another way. One can use an analytical continuation of the hypergeometric function [11], namely

$$
\begin{aligned}
& { }_{2} F_{1}\left(-\frac{1}{2}, s-1 ; \frac{1}{2} ;-\left(\frac{n \nu}{b}\right)^{2}\right)=\frac{n \nu}{b} \frac{\Gamma(1 / 2) \Gamma(s-1 / 2)}{\Gamma(s-1)} \\
+ & \frac{\Gamma(1 / 2) \Gamma(1 / 2-s)}{\Gamma(-1 / 2) \Gamma(3 / 2-s)}\left(1+\left(\frac{n \nu}{b}\right)^{2}\right)^{1-s}{ }_{2} F_{1}\left(1, s-1 ; s+\frac{1}{2} ; \frac{1}{1+\left(\frac{n \nu}{b}\right)^{2}}\right) .
\end{aligned}
$$

The first term in the rhs. cancels the second, divergent term in the sum (23). Next, one can use power series expansion for the hypergeometric function because its argument $1 /\left(1+(n \nu / b)^{2}\right)$ is always smaller then unity. The result will be the same as that obtained above by a longer calculation.

The series $Z(p, q)$ in (22) can be expressed in terms of the Epstein - Hurwitz zeta function [12

$$
\zeta_{E H}(r, c)=\sum_{n=1}^{\infty}\left(n^{2}+c^{2}\right)^{-r}
$$

A known, quickly convergent expansion for large values of the parameter $c$ is

$$
\zeta_{E H}(r, c)=-\frac{c^{-2 r}}{2}+\frac{\sqrt{\pi} \Gamma(r-1 / 2)}{2 \Gamma(r)} c^{-2 r+1}+\frac{2 \pi^{r} c^{-r+1 / 2}}{\Gamma(r)} \sum_{n=1}^{\infty} n^{r-1 / 2} K_{r-1 / 2}(2 \pi n c) .
$$

For small $c$ it holds

$$
\zeta_{E H}(r, c)=\sum_{n=0}^{\infty}(-1)^{n} \frac{\Gamma(n+r)}{\Gamma(r) n !} c^{2 r} \zeta_{R}(2 r+2 n) .
$$

For integer $r \geq 1$ the zeta function can be expressed in terms of elementary functions. So we get the relevant part of the ground state energy in the form

$$
\begin{aligned}
E_{0}^{\text {thin }}(s)= & \left(\frac{M}{m}\right)^{2 s} \frac{1}{8 \pi}\left(-\frac{2 \pi R^{2}}{3 \nu} m^{3}-\frac{\pi^{3 / 2} R m^{2} \Gamma(s-1)}{\nu \Gamma\left(s-\frac{1}{2}\right)}+\frac{\pi}{3}\left(\nu+\frac{1}{\nu}\right) m\right. \\
& \left.+\frac{\pi^{3 / 2} \Gamma(s)}{32 R \nu \Gamma\left(s-\frac{1}{2}\right)}\right)+\frac{1}{R} O\left(\frac{1}{R m}\right)
\end{aligned}
$$

dropping contributions proportional to $\exp (-R m)$. By comparing this formula with $E_{0}^{\text {div }}(7)$ we can read off the heat kernel coefficients

$$
B_{0}=\frac{\pi R^{2}}{\nu}, B_{1 / 2}=-\frac{\pi^{3 / 2} R}{\nu}, B_{1}=\frac{\pi}{3}\left(\nu+\frac{1}{\nu}\right), B_{3 / 2}=\frac{\pi^{3 / 2}}{32 R \nu} .
$$

Now, taking into account the general structure

$$
B_{r}=\int_{\partial V} c_{r} d S+\int_{V} a_{r} d V
$$


of the coefficients we represent $B_{1}$ as

$$
B_{1}=\frac{2 \pi}{3 \nu}+\frac{\pi}{3}\left(\nu-\frac{1}{\nu}\right) .
$$

In this representation, the first term in $B_{1}$ and all other coefficients are seen to be the result of the boundary at $r=R$. In fact, they are known [13]. The second term in $B_{1}$ is independent on that boundary. It is known as the topological Kac term [7] and is a result of the conical singularity.

Using these coefficients, by means of Eq.(17), we can define $E_{0}^{\text {div }}$ and the renormalized ground state energy $E_{0}^{\text {ren }}(\mathbb{8})$. Now we observe, that all contributions in (26) except for the topological Kac term are due the the boundary at $r=R$. Leaving them aside, only the Kac term remains. It must be included into $E_{0}^{\text {div }}$ and we get

$$
E_{0}^{\mathrm{ren}}=0
$$

for the genuine contributions of the string, or equally in the limit $R \rightarrow \infty$. Let us remark, that this result holds also in $(3+1)$ and higher dimensions as can be inferred from dimensional reasons. The parameters entering the problem are the mass density of the string which enters together with the graviational constant to form a dimensionless combination, expressed by the angle deficite, for example and the mass of the quantum field. In the case of a string with zero radius there is no further dimensional parameter on which the renormalized ground state energy might depend. As the ground state energy has the dimension of a inverse length it might be proportional to the mass but such terms had been subtracted in the renormalization process. Note that this discussion does not apply to the case of a string with finite radius because this radius is the additional dimensional parameter allowing in general for a nontrivial renormalised ground state energy.

\section{Cosmic string with finite thickness}

We use the metric given by Eq. (13), (14). Again, the coordinate $z$ will be dropped because we work in $(2+1)$ dimensions. The curvature scalar is

$$
\mathrm{R}=-\frac{2 \epsilon^{2}}{\rho_{0}^{2}}
$$

and we allow for $\xi \neq \frac{1}{6}$. By means of the ansatz $(n=0, \pm 1, \pm 2, \ldots)$

$$
\Phi=e^{i n \varphi} g^{-1 / 4} \mathcal{R}=e^{i n \varphi}\left\{\begin{array}{l}
\mathcal{R}_{\text {in }}(\rho) / \sqrt{\frac{\rho_{0}}{\epsilon} \sin \left(\frac{\epsilon \rho}{\rho_{0}}\right)}, \rho \in\left[0, \rho_{0}\right] \\
\mathcal{R}_{\text {out }}(r) / \sqrt{\frac{r}{\nu}}, r \in\left[r_{0}, \infty\right),
\end{array}\right.
$$

we arrive at the equations

$$
\left\{\frac{d^{2}}{d \rho^{2}}-\frac{\epsilon^{2}\left[n^{2}-1 / 4\right]}{\rho_{0}^{2} \sin ^{2}\left(\epsilon \rho / \rho_{0}\right)}+\frac{\epsilon^{2}}{4 \rho_{0}^{2}}(1-8 \xi)+\lambda^{2}\right\} \mathcal{R}_{i n}=0, \rho \in\left[0, \rho_{0}\right]
$$




$$
\left\{\frac{d^{2}}{d r^{2}}-\frac{n^{2} \nu^{2}-1 / 4}{r^{2}}+\lambda^{2}\right\} \mathcal{R}_{\text {out }}=0, r \in\left[r_{0}, \infty\right)
$$

for the radial functions. Both this equations may be solved exactly. Indeed, the solution of the radial equation outside of the string (34) can be written as

$$
\mathcal{R}_{\text {out }}=\frac{i}{2}\left[f_{n}(\lambda) H_{n \nu}^{-}(\lambda r)-f_{n}^{*}(\lambda) H_{n \nu}^{+}(\lambda r)\right],
$$

where $f_{n}$ is the Jost function and

$$
H_{n \nu}^{ \pm}(\lambda r)= \pm i \sqrt{\frac{\pi \lambda r}{2}} H_{n \nu}^{(1),(2)}(\lambda r)
$$

are combinations of the Hankel functions. The solution regular at the origin of the radial equation (33) inside the string is

$$
\mathcal{R}_{\text {in }}=e^{i \frac{\pi}{2} n(\nu-1)} \sqrt{\frac{\pi}{2} \sin \frac{\epsilon \rho}{\rho_{0}}}\left(\frac{\lambda \rho_{0}}{\epsilon}\right)^{n+1 / 2} \mathrm{P}_{\alpha}^{-n}\left[\cos \frac{\epsilon \rho}{\rho_{0}}\right]
$$

with

$$
\alpha=-\frac{1}{2}+\frac{1}{2} \sqrt{1+\frac{4 \lambda^{2} \rho_{0}^{2}}{\epsilon^{2}}-8 \xi}
$$

and $\mathrm{P}_{\alpha}^{n}$ is the Legendre function. These solutions must obey the matching conditions

$$
\mathcal{R}_{\text {in }}\left(\rho_{0}\right)=\mathcal{R}_{\text {out }}\left(r_{0}\right), \mathcal{R}_{\text {in }}^{\prime}\left(\rho_{0}\right)=\mathcal{R}_{\text {out }}^{\prime}\left(r_{0}\right)
$$

on the surface of the string. From this we get the following formula for the Jost function

$$
\begin{aligned}
f_{n}(\lambda)= & -e^{i \frac{\pi}{2} n(\nu-1)} \frac{i \pi}{2} \frac{\sin \epsilon}{\sqrt{\cos \epsilon}}\left(\frac{\lambda \rho_{0}}{\epsilon}\right)^{n+1} \\
& \times\left\{H_{n \nu}^{(1)^{\prime}}\left(\lambda r_{0}\right) \mathrm{P}_{\alpha}^{-n}[\cos \epsilon]+H_{n \nu}^{(1)}\left(\lambda r_{0}\right) \mathrm{P}_{\alpha}^{-n^{\prime}}[\cos \epsilon] \frac{\epsilon \sin \epsilon}{\lambda \rho_{0}}\right\} .
\end{aligned}
$$

Here the primes denote derivatives with respect to the arguments. Taken on the imaginary axis this Jost function reads

$$
\begin{aligned}
f_{n}(i k)= & -\frac{\sin \epsilon}{\sqrt{\cos \epsilon}}\left(\frac{k \rho_{0}}{\epsilon}\right)^{n+1} \\
& \times\left\{K_{n \nu}^{\prime}\left(k r_{0}\right) \mathrm{P}_{\alpha}^{-n}[\cos \epsilon]+K_{n \nu}\left(k r_{0}\right) \mathrm{P}_{\alpha}^{-n^{\prime}}[\cos \epsilon] \frac{\epsilon \sin \epsilon}{k \rho_{0}}\right\} .
\end{aligned}
$$

Note that this function doesn't have zeros for $k \in[0, \infty)$, i.e., there are no bound states. This can be checked by inspection of eq. (37). The Jost function has the following asymptotics for large resp. small $k$ :

$$
\begin{aligned}
f_{n}(i k)_{k \rightarrow \infty} & \sim \exp \left\{-k \rho_{0}\left(\frac{r_{0}}{\rho_{0}}-1\right)\right\} \\
f_{n}(i k)_{k \rightarrow 0} & \sim k^{-n(\nu-1)}, f_{0}(i k)_{k \rightarrow 0} \sim-\ln k .
\end{aligned}
$$


Using the formula

$$
\lim _{t \rightarrow \infty} t^{n} \mathrm{P}_{t}^{-n}\left[\cos \frac{x}{t}\right]=J_{n}(x),
$$

the Minkowskian limit $(\nu \rightarrow 1)$ for the Jost function

$$
\lim _{\nu \rightarrow 1} f_{n}(\lambda)=1
$$

can be checked.

Proceeding as it was done in the case of the infinitely thin string we obtain the following expression for the ground state energy in $(2+1)$ dimensions

$$
\begin{aligned}
E_{0}= & -M^{2 s} \frac{\cos \pi s}{2 \pi} \sum_{n=0}^{+\infty} d_{n} \int_{m}^{\infty} d k\left[k^{2}-m^{2}\right]^{1 / 2-s} \\
& \times \frac{\partial}{\partial k} \ln \left\{k^{-n}\left[f_{n}(i k) H_{n \nu}^{-}(i k R)-f_{n}^{*}(i k) H_{n \nu}^{+}(i k R)\right]\right\}
\end{aligned}
$$

and, using

$$
K_{\mu}\left(z \mathrm{e}^{-i \pi}\right)=\mathrm{e}^{-i \mu z} K_{\mu}(z)-i \pi I_{\mu}(z)
$$

after obvious rearrangements we get

$$
\begin{aligned}
E_{0}= & -M^{2 s} \frac{\cos \pi s}{2 \pi} \sum_{n=0}^{+\infty} d_{n} \int_{m}^{\infty} d k\left[k^{2}-m^{2}\right]^{1 / 2-s} \\
& \times \frac{\partial}{\partial k} \ln \left\{k^{-n}\left[f_{n}(i k) I_{n \nu}(k R)-\widetilde{f}_{n}(i k) K_{n \nu}(k R)\right]\right\} \\
= & -M^{2 s} \frac{\cos \pi s}{2 \pi} \sum_{n=0}^{+\infty} d_{n} \int_{m}^{\infty} d k\left[k^{2}-m^{2}\right]^{1 / 2-s} \frac{\partial}{\partial k} \ln k^{-n} f_{n}(i k) I_{n \nu}(k R) \\
& -M^{2 s} \frac{\cos \pi s}{2 \pi} \sum_{n=0}^{+\infty} d_{n} \int_{m}^{\infty} d k\left[k^{2}-m^{2}\right]^{1 / 2-s} \frac{\partial}{\partial k} \ln \left[1-\frac{\widetilde{f}_{n}(i k)}{f_{n}(i k)} \frac{K_{n \nu}(k R)}{I_{n \nu}(k R)}\right] .
\end{aligned}
$$

Now the contribution of the last line is exponentially small for $R \rightarrow \infty$. The contribution of the preceeding line can be written as the sum

$$
E_{0}=E_{0}^{\text {thin }}+E_{0}^{\text {int }}
$$

of the contribution $E_{0}^{\text {thin }}$ (20) of the infinitely thin string considered in the preceeding section and the contribution of the interior structure of the string

$$
E_{0}^{\mathrm{int}}=-M^{2 s} \frac{\cos \pi s}{2 \pi} \sum_{n=0}^{+\infty} d_{n} \int_{m}^{\infty} d k\left[k^{2}-m^{2}\right]^{1 / 2-s} \frac{\partial}{\partial k} \ln k^{n(\nu-1)} f_{n}(i k) .
$$

After the work done in section 3 it is just this contribution which remains to be calculated. 


\section{Approximation of small angle deficit}

To calculate $E_{0}^{\text {int }}(42)$ and the corresponding renormalised ground state energy the analytic continuation in $s$ to $s=0$ must be performed. To this end the knowledge of the uniform asymptotic expansion of the Jost function $f_{n}(i k)$ (37) for $n \rightarrow \infty, k \rightarrow \infty, n / k$ fixed is required. Now, althought this Jost function is known explicitely in terms of Bessel functions and the Legendre function, this task is not easy to perform. The point is that the asymptotic for $\alpha \rightarrow \infty$ and $n \rightarrow \infty$ of the Legendre function $P_{\alpha}^{-n}$ is quite complicated to handle. Therefore we restrict ourselfs to the easier tractable case of a small angle deficit resp. mass density of the string, i.e., to the case $\epsilon \ll 1$. Than in the radial equation for the interior of the string

$$
\left\{\frac{d^{2}}{d \rho^{2}}-\frac{n^{2}-1 / 4}{\rho^{2}}+\lambda^{2}-U(\rho)\right\} \mathcal{R}_{i n}=0, \rho \in\left[0, \rho_{0}\right],
$$

with the potential (using (31))

$$
U(\rho)=\frac{1}{\rho^{2}}\left(\left(n^{2}-\frac{1}{4}\right)\left(\frac{\theta^{2}}{\sin ^{2} \theta}-1\right)+\theta^{2}\left(2 \xi-\frac{1}{4}\right)\right)
$$

$\left(\theta=\epsilon \rho / \rho_{0}\right)$ we approximate

$$
U(\rho)=U_{0}+O\left(\epsilon^{4}\right)
$$

with

$$
U_{0}=\frac{e^{2}}{3 \rho_{0}^{2}}\left(n^{2}+6 \xi-1\right) .
$$

By this, Eq. (43) can be solved in terms of Bessel functions

$$
\mathcal{R}_{\text {in }}=e^{i \frac{\pi n}{2}(\nu-1)}\left(\frac{\lambda}{\lambda_{n}}\right)^{n+1 / 2} \sqrt{\frac{\pi}{2} \lambda_{n} \rho} J_{n}\left(\lambda_{n} \rho\right),
$$

$\left(\lambda_{n}=\sqrt{\lambda^{2}-U_{0}}\right)$ and the corresponding Jost function reads for small $\epsilon$

$$
\begin{aligned}
f_{n}^{\mathrm{se}}(\lambda)= & i \frac{\pi}{2} e^{i \frac{\pi n}{2}(\nu-1)}\left(\frac{\lambda}{\lambda_{n}}\right)^{n}\left\{\frac{1}{2}\left[\sqrt{\frac{r_{0}}{\rho_{0}}}-\sqrt{\frac{\rho_{0}}{r_{0}}}\right] J_{n}\left(\lambda_{n} \rho_{0}\right) H_{n \nu}^{(1)}\left(\lambda r_{0}\right)\right. \\
& \left.+\sqrt{r_{0} \rho_{0}}\left[\lambda_{n} J_{n}^{\prime}\left(\lambda_{n} \rho_{0}\right) H_{n \nu}^{(1)}\left(\lambda r_{0}\right)-\lambda J_{n}\left(\lambda_{n} \rho_{0}\right) H_{n \nu}^{(1)^{\prime}}\left(\lambda r_{0}\right)\right]\right\} .
\end{aligned}
$$

On the imaginary axis we get

$$
\begin{aligned}
f_{n}^{\mathrm{se}}(i k)= & \left(\frac{k}{k_{n}}\right)^{n}\left\{\frac{1}{2}\left[\sqrt{\frac{r_{0}}{\rho_{0}}}-\sqrt{\frac{\rho_{0}}{r_{0}}}\right] I_{n}\left(k_{n} \rho_{0}\right) K_{n \nu}\left(k r_{0}\right)\right. \\
& \left.+\sqrt{\frac{r_{0}}{\rho_{0}}}\left[k_{n} \rho_{0} I_{n}^{\prime}\left(k_{n} \rho_{0}\right) K_{n \nu}\left(k r_{0}\right)-k \rho_{0} I_{n}\left(k_{n} \rho_{0}\right) K_{n \nu}^{\prime}\left(k r_{0}\right)\right]\right\},
\end{aligned}
$$


with $r_{0} / \rho_{0}=\tan \epsilon / \epsilon$ and $k_{n}=\sqrt{k^{2}+\frac{\epsilon^{2}}{3 \rho_{0}^{2}}\left(n^{2}+6 \xi-1\right)}$. It is easy to verify that it obeys the limits (38) and (40).

Note that the approximate potential $U$ (44) is constant and that $U_{0}$ may take negative values. Therefore bound states could occur. However, due to the conical structure of the exteriour part of the space (see $\nu$ in the index of the Hankel functions) and the corresponding relations between the parameters, in fact no bound states occur. Correspondingly, it can be shown that the Jost function (45) doesnt have zeros for $k \in[0, \infty)$.

Now we insert this approximate Jost function into $E_{0}^{\text {int }}$ (42) and have to perform the analytic continuation in $s$ to $s=0$. For this reason we use the uniform asymptotic expansion of (45) which can now be obtained by simply inserting the corresponding expansion (21) of the Bessel functions. We define

$$
f_{n}^{\mathrm{se}, \text { as }}(i k)=\frac{z^{n \nu}}{y^{n}} e^{-n \nu \eta\left(z_{\nu}\right)+n \eta(y)}\left(1+\epsilon^{2} \frac{t}{24 n}\right)
$$

with

$$
\begin{aligned}
z & =\frac{k \rho_{0}}{n}, z_{\nu}=\frac{k r_{0}}{n \nu}, t=\left(1+z^{2}\right)^{-1 / 2} \\
\eta(z) & =\sqrt{1+z^{2}}+\ln \frac{z}{1+\sqrt{1+z^{2}}}, y=\sqrt{z^{2}+\frac{\epsilon^{2}}{3}-\frac{\epsilon^{2}}{n^{2}} \frac{1-6 \xi}{3}} .
\end{aligned}
$$

In general, the expansion (46) must include all terms up to $n^{-2}$. But that contribution doesn't appear (together with $n^{-4}, n^{-6}, \ldots$ ). Next we divide the expression for $E_{0}^{\text {int }}$ into two parts

$$
E_{0}^{\text {int }}=E_{0, \text { as }}^{\text {int }}+E_{0, \text { fin }}^{\text {int }} .
$$

simply subtracting and adding $\ln k^{n(\nu-1)} f_{n}^{\text {se, as in }}$ (42). Here

$$
E_{0, \mathrm{as}}^{\mathrm{int}}=-M^{2 s} \frac{\cos \pi s}{2 \pi} \sum_{n=0}^{+\infty} d_{n} \int_{m}^{\infty} d k\left[k^{2}-m^{2}\right]^{1 / 2-s} \frac{\partial}{\partial k} \ln k^{n(\nu-1)} f_{n}^{a s}(i k),
$$

is called the 'asymptotic' part which still requires the analytic continuation to be done and

$$
E_{0, \text { fin }}^{\text {int }}=\frac{-1}{2 \pi} \sum_{n=0}^{+\infty} d_{n} \int_{m}^{\infty} d k \sqrt{k^{2}-m^{2}} \frac{\partial}{\partial k}\left[\ln k^{n(\nu-1)} f_{n}(i k)-\ln k^{n(\nu-1)} f_{n}^{a s}(i k)\right]
$$

is called the 'finite' part. In it the analytic continuation could be performed under the sign of the integral and the sum because they are convergent.

In $E_{0 \text {,as }}^{\text {int }}(48)$ the integrand can be expanded in powers of $\epsilon$. Than the integration over $k$ and the summation over $n$ can be carried out explicitely using the 
same method as in section 3 . We obtain

$$
\begin{aligned}
\left.E_{0, \text { as }}^{\text {int }}\right|_{s \rightarrow 0}= & \frac{\epsilon^{2}}{2 \pi \rho_{0}}\left\{\frac{\pi}{24} \beta^{3}-\left[\frac{\pi}{12}-\frac{1-6 \xi}{12} \pi\right] \beta-\left[\frac{1}{720}+\frac{1-6 \xi}{72}\right] \frac{\pi}{\beta}\right. \\
& +\left[\frac{1}{6} \beta^{2}-\frac{1}{24}+\frac{1-6 \xi}{6}\right] \ln \left(1-e^{-2 \pi \beta}\right) \\
& \left.-\frac{\pi}{3} \beta^{2}\left[3 \widetilde{Q}_{1}(\beta)-3 \widetilde{Q}_{2}(\beta)+\widetilde{Q}_{3}(\beta)\right]-\frac{1-6 \xi}{3} \pi \widetilde{Q}_{1}(\beta)\right\},
\end{aligned}
$$

with the notation $\beta=m \rho_{0}$ and $\tilde{Q}_{a}$ defined by (25). We remark that $\tilde{Q}_{a}(\beta)$ are exponentially decreasing for $\beta \rightarrow \infty$.

At this place we can determine the heat kernel coefficients for the thick string. We have to consider the asymptotic expansion of $E_{0}^{\text {int }}$ for large $m$. The nondecreasing contributions may be contained only in $E_{0 \text {,as }}^{\text {int }} 48$ and can be read off from Eqs. (50) and (7). They are

$$
B_{0}^{\text {int }}=-\epsilon^{2} \frac{\pi \rho_{0}^{2}}{4}, \quad B_{1}^{\text {int }}=-2 \pi \xi \epsilon^{2} .
$$

Note that the coefficient $B_{1 / 2}$ is zero. This was to be expected because the background is smooth enougth not to allow for boundary dependent coeficients in this order. Also, the coefficient $B_{3 / 2}$ is zero. This is in the given order in $\epsilon$ and follows simply from dimensional reasons. In higher orders in $\epsilon$ it may be nonzero like further coefficients of higher half integer order.

Now, by means of (41) we have to take the contributions to the heat kernel coefficients of the infinitely thin string (27) and that of the 'interior', Eq. (51) together. These coefficients can be compared with that following from the general formulas. For instance, from

$$
\begin{aligned}
B_{0}=\int_{V} \mathrm{~d} V & =2 \pi \int_{0}^{\rho_{0}} \frac{\rho_{0}}{\epsilon} \sin \frac{\epsilon \rho}{\rho_{0}} d \rho+\frac{2 \pi}{\nu} \int_{r_{0}}^{R} r \mathrm{~d} r \\
& =\frac{\pi R^{2}}{\nu}-\frac{\epsilon^{2} \pi \rho_{0}^{2}}{4}+O\left(\epsilon^{4}\right),
\end{aligned}
$$

we obtain the boundary dependent contribution (cf. Eq. (27)) and $B_{0}^{\text {int }}$ (51). For the coefficient $B_{1}$ we have

$$
B_{1}=\frac{1-6 \xi}{6} \int_{V} \operatorname{Rd} V=\frac{1-6 \xi}{3} \epsilon^{2} \pi+O\left(\epsilon^{4}\right) .
$$

Now, in the given approximation it holds

$$
\frac{\pi}{3}\left(\nu-\frac{1}{\nu}\right)=\frac{\pi}{3} \epsilon^{2}+O\left(\epsilon^{4}\right) .
$$


Therefore, from $B_{1}$ (27) resp. (29) of the thin string and $B_{1}^{\text {int }}$ (51) we get

$$
B_{1}=\frac{\pi}{3}\left(\nu+\frac{1}{\nu}\right)-2 \pi \xi \epsilon^{2}=\frac{2 \pi}{3 \nu}+\epsilon^{2} \pi \frac{1-6 \xi}{3}+O\left(\epsilon^{4}\right) .
$$

We note that the Kac term disappeared. The first term in the last line is due to the boundary at $r=R$ and the second is the genuine contribution of the thick string. It vanishes in case of a conformal coupling.

To proceed, we define $E_{0}^{\text {ren }}$ by means of (\$) using the coefficients $B_{0}$ (52) and $B_{1}$ (53) in the definition of $E_{0}^{\text {div }}$ (7). Note that the coefficients with half integer numbers are pure boundary dependent contributions resulting from the boundary conditions at $r=R$.

In fact, the renormalization in $E_{0}^{\text {int }}$ is reduced to dropping the nondecreasing for $m \rightarrow \infty$ contributions in $E_{0 \text {,as }}^{\text {int }}$. Therefore we obtain for the complete renormalized ground state energy

$$
\begin{aligned}
E_{0}^{\mathrm{ren}}= & \frac{\epsilon^{2}}{2 \pi \rho_{0}}\left\{\left[\frac{1}{6} \beta^{2}-\frac{1}{24}+\frac{1-6 \xi}{6}\right] \ln \left(1-e^{-2 \pi \beta}\right)\right. \\
& -\frac{\pi}{3} \beta^{2}\left[3 \widetilde{Q}_{1}(\beta)-3 \widetilde{Q}_{2}(\beta)+\widetilde{Q}_{3}(\beta)\right]-\frac{1-6 \xi}{3} \pi \widetilde{Q}_{1}(\beta) \\
& \left.-\sum_{n=0}^{\infty} d_{n} \int_{\beta}^{\infty} d x \sqrt{x^{2}-\beta^{2}} \frac{\partial}{\partial x} F_{n}-\left[\frac{1}{720}+\frac{1-6 \xi}{6}\right] \frac{\pi}{\beta}\right\} .
\end{aligned}
$$

Here, the notation

$$
F_{n}=\frac{\ln f_{n}(i x)-\ln f_{n}^{a s}(i x)}{\epsilon^{2}}
$$

is introduced.

Some further work is necesary. In $F_{n}$ the Jost functions have still to be expanded for small $\epsilon$. For $f_{n}(i k)$ this can be done using the formula [11]

$$
\left.\frac{\partial K_{p}(x)}{\partial p}\right|_{p=n}=\frac{n !}{2}\left(\frac{x}{2}\right)^{-n} \sum_{l=0}^{n-1}\left(\frac{x}{2}\right)^{n} \frac{K_{l}(x)}{(n-l) l !},
$$

for $f_{n}^{\text {as }}(i k)$ this expansion is a simple task. Finally we obtain

$$
\begin{aligned}
F_{\left.n\right|_{\epsilon=0}}= & \left(\frac{(n+1)^{2}}{6}-\frac{x^{2}}{3}\right) I_{n} K_{n}+\left(\frac{n^{2}}{6}-\frac{x^{2}}{3}\right) I_{n+1} K_{n-1}+\frac{x}{3} I_{n+1} K_{n} \\
& +\frac{n n !}{4} \sum_{l=0}^{n-1}\left(\frac{x}{2}\right)^{l-n} \frac{x\left(I_{n+1} K_{l}+I_{n} K_{l+1}\right)}{(n-l) l !}+\frac{n}{2}\left(K_{0} n ! I_{n}\left(\frac{2}{x}\right)^{n}+\ln x\right) \\
& +\left.\frac{n n !}{2} \sum_{l=1}^{n-1}\left(\frac{x}{2}\right)^{l-n} \frac{I_{n} K_{l}}{l !}\right|_{n \geq 2}+\frac{n}{3} \sqrt{1+\frac{x^{2}}{n^{2}}}-\frac{n}{6} \frac{1}{1+\sqrt{1+\frac{x^{2}}{n^{2}}}} \\
& -\frac{n}{2} \ln \left(1+\sqrt{1+\frac{x^{2}}{n^{2}}}\right)-\frac{1}{24 n} \frac{1}{\sqrt{1+\frac{x^{2}}{n^{2}}}}
\end{aligned}
$$




$$
-\frac{1-6 \xi}{6}\left[I_{n} K_{n}+I_{n+1} K_{n-1}-\frac{1}{n} \frac{1}{1+\sqrt{1+\frac{x^{2}}{n^{2}}}}\right] .
$$

This expression has to be used in $E_{0}^{\text {ren }}$ (55).

In writing

$$
E_{0}^{\mathrm{ren}}=\frac{\epsilon^{2}}{2 \pi \rho_{0}} G(\beta)+O\left(\epsilon^{4}\right)
$$

it is in fact $G(\beta)$, a function of one variable, which must be calculated. We did this task numerically. After a carefully examination we came to the result

$$
G(\beta)=0,
$$

for arbitrary $\xi$; the details are given in the appendix.

\section{Conclusions}

In the preceeding sections we worked out methods suited for the calculation of the ground state energy of a massive scalar field in the background of a cosmic string. The main emphasis was on a string of finite thickness. We used the standard renormalization scheme, i.e., we calculated and subtracted the contributions of the first few heat kernel coefficients. Thereby the normalisation condition, stating that the renormalised ground state energy must vanish for a large mass of the quantum field, is imposed.

As a part of these calculations we first considered the infinitely thin string in detail. Using explicit formulas we reobtained the known heat kernel coefficients. These are the coefficients due to the boundary of a large cylinder at $r=R$, which was introduced in order to render the eigenvalues discrete, and the topological Kac term. In the sense of the renormalisation used we got the result that the renormalised ground state energy of the pure string, i.e., when removing the outer boundary $(R \rightarrow \infty)$, is zero.

Then the same problem is formulated for a string with finite thickness. However, the complete calculation suffers still from mathematical difficulties. Therefore the approximation of a small mass density $\epsilon$ of the string was introduced. Having in mind the smallness of $\epsilon \sim 10^{-3}$ in cosmological applications, this is at once physically motivated.

We note that in this approximation an alternative calculation should be possible, namely the use of a perturbation theory in the mass density as it was done for the calculation of the Casimir force between two cosmic strings in [15].

In this approximation of a small mass density, in order $\epsilon^{2}$, first the heat kernel coefficients were calculated. They are checked to coincide with that following from general formulas. We remark that the Kac term disappeares and that for a conformal coupling $(\xi=1 / 6)$ there are no counterterms required besides that 
which follow from the boundary at $r=R$. From this it is clear that the Kac term is due to the singular behaviour of the metric of the thin string at the origin. This can be understood from another point of view too. Consider the vacuum expectation value of the energy density in the background of the thin string. For dimensional reasons it behaves like $r^{-2}$ near the string and, therefore, cannot be integrated over $r$ near the origin. Now, if introducing a suitable regularisation, zeta functional regularisation for instance, it becomes possible to integrate over $r$. As a result, when removing the regularisation, an additional divergence occurs which is just the Kac term.

After performing the renormalisation we calculated numerically the ground state energy in the background of the thick cosmic string in order $\epsilon^{2}$ of a small mass density. The result is zero with the reasonable precision of $10^{-7}$ independently of the parameter $\xi$. Thereby a nontrivial compensation between different contributions occured.

This result that the vacuum of a scalar field is not disturbed by a cosmic string is quite remarkable and seems not to have analogues in other configurations. For instance, we do not see any symmetry or invariance arguments for this result although they should be there.

Perhaps, there is some relation to the result of Brevik and Jenesen 16] indicating the absence of particle production in the formation of a cosmic string.

Further work is necessary. For instance, the result should be extended to the $(3+1)$ dimensional case, to higher spin fields and to mass densities which are not small.

\section{Acknowledgement}

The authors would like to thank K. Kirsten and D. Fursaev for discussions.

NK is supported in part by Russian Found of Fundamental Research (grant No 97-02-16318).

\section{Appendix}

Here we consider the analytical and numerical analysis of the function $G(\beta)$ defined in Eq. (57)

$$
\begin{aligned}
G(\beta)= & {\left[\frac{1}{6} \beta^{2}-\frac{1}{24}+\frac{1-6 \xi}{6}\right] \ln \left(1-e^{-2 \pi \beta}\right)-\frac{\pi}{3} \beta^{2}\left[3 \widetilde{Q}_{1}(\beta)-3 \widetilde{Q}_{2}(\beta)+\widetilde{Q}_{3}(\beta)\right] } \\
& -\frac{1-6 \xi}{3} \pi \widetilde{Q}_{1}(\beta)-\sum_{n=0}^{\infty} d_{n} \int_{\beta}^{\infty} d x \sqrt{x^{2}-\beta^{2}} \frac{\partial F_{n}}{\partial x}-\left[\frac{1}{720}+\frac{1-6 \xi}{72}\right] \frac{\pi}{\beta} .(58)
\end{aligned}
$$

Obviously we have $G(\beta)_{\beta \rightarrow \infty} \rightarrow 0$ and the domain of interest is the neighborhood of zero: $\beta \sim 0$. For numerical simulations the above formula is more suitable 
for $\beta \geq 1$. In the opposite case, $\beta<1$, there exist at first sight a logarithmic singularity for small $\beta: \ln (1-\exp (-2 \pi \beta)) \sim \ln 2 \pi \beta$. But this singularity is canceled with that in the contribution of $(n=0)$ in the series in (58). For numerical calculations it is more suitable to cancel this singularity in manifest form. For this reason we divide this term into two parts,

$$
\int_{\beta}^{\infty} d x \sqrt{x^{2}-\beta^{2}} \frac{\partial}{\partial x} F_{0}=\int_{\beta}^{1} d x \sqrt{x^{2}-\beta^{2}} \frac{\partial}{\partial x} F_{0}+\int_{1}^{\infty} d x \sqrt{x^{2}-\beta^{2}} \frac{\partial}{\partial x} F_{0},
$$

where

$$
\begin{aligned}
F_{0} & =\Phi_{0}-\Phi_{0}^{a s}, \\
\Phi_{0} & =\frac{1-x^{2}}{6} I_{0} K_{0}-\frac{x^{2}}{3} I_{1} K_{1}-\frac{x^{2}}{6} I_{0} K_{2}-\frac{1-6 \xi}{6}\left(I_{0} K_{0}+I_{1} K_{1}\right), \\
\Phi_{0}^{a s} & =-\frac{x}{3}+\frac{1}{24 x}-\frac{1-6 \xi}{6 x} .
\end{aligned}
$$

The integral

$$
\int_{\beta}^{1} d x \sqrt{x^{2}-\beta^{2}} \frac{\partial}{\partial x} \Phi_{0}^{a s}
$$

may be found in manifest form. Thereby we arrive at the following expression for the case $\beta<1$

$$
\begin{aligned}
G(\beta)= & {\left[\frac{1}{6} \beta^{2}-\frac{1}{24}+\frac{1-6 \xi}{6}\right] \ln \left[\frac{1-e^{-2 \pi \beta}}{\beta}\left(1+\sqrt{\left.1-\beta^{2}\right)}\right]\right.} \\
& -\frac{\pi}{3} \beta^{2}\left[3 Q_{1}(\beta)-3 Q_{2}(\beta)+Q_{3}(\beta)\right]-\frac{1-6 \xi}{3} \pi Q_{1}(\beta) \\
& +\frac{\pi \beta}{24}+\zeta_{R}^{\prime}(-2)-\left[\frac{1}{8}+\frac{1-6 \xi}{6}\right] \sqrt{1-\beta^{2}}-\int_{\beta}^{1} d x \sqrt{x^{2}-\beta^{2}} \frac{\partial}{\partial x} \Phi_{0} \\
& -\int_{1}^{\infty} d x \sqrt{x^{2}-\beta^{2}} \frac{\partial}{\partial x} F_{0}-\sum_{n=1}^{\infty} 2 \int_{\beta}^{\infty} d x \sqrt{x^{2}-\beta^{2}} \frac{\partial}{\partial x} F_{n} .
\end{aligned}
$$

with the notation

$$
Q_{n}(\beta)=\frac{1}{\beta^{n}} \int_{0}^{\beta} \frac{d t t^{n}}{e^{2 \pi t}-1} .
$$

It is necessary to stress that formula (61) is only a different representation of $G(\beta)$ (58) which we made in order to avoid the logarithmic contribution in individual terms. Next we observe that the series over $n$ is slowly convergent and quite a large number of terms must be taken into account

Now let us consider some analytical properties of $G(\beta)$. This function doesn't have a linear term in the expansion for small $\beta$,

$$
G(\beta)=G(0)+O\left(\beta^{2}\right) .
$$


In order to argue this statement let's consider the part in (61) which contains the integral

$$
\begin{aligned}
D(\beta) & =\int_{\beta}^{1} d x \sqrt{x^{2}-\beta^{2}} \frac{\partial \Phi_{0}}{\partial x}+\int_{1}^{\infty} d x \sqrt{x^{2}-\beta^{2}} \frac{\partial F_{0}}{\partial x} \\
& +\sum_{n=1}^{\infty} 2 \int_{\beta}^{\infty} d x \sqrt{x^{2}-\beta^{2}} \frac{\partial F_{n}}{\partial x} .
\end{aligned}
$$

The function $\Phi_{0}$ has the following expansion for small $x$ (using the power series expansion of the Bessel functions)

$$
\Phi_{0}=\ln \frac{x}{2} \sum_{k=0}^{\infty} C_{k} x^{2 k}+\sum_{k=0}^{\infty} \widetilde{C}_{k} x^{2 k} .
$$

It is only the zeroth term in the first sum, $C_{0} \ln x / 2$, which deliveres a linear contribution to the first integral in (63). All other terms contribute higher powers in $\beta$. Thus

$$
\int_{\beta}^{1} d x \sqrt{x^{2}-\beta^{2}} \frac{\partial \Phi_{0}}{\partial x}=\int_{0}^{1} d x x \frac{\partial \Phi_{0}}{\partial x}-\frac{\pi}{2} C_{0} \beta+O\left(\beta^{2}\right)
$$

with $C_{0}=(1-6 \xi) / 6-1 / 6=-\xi$.

In the second integral in (63) we can expand the integrand for $\beta^{2} / x^{2} \ll 1$ obtain

$$
\int_{1}^{\infty} d x \sqrt{x^{2}-\beta^{2}} \frac{\partial F_{0}}{\partial x}=\int_{1}^{\infty} d x x \frac{\partial F_{0}}{\partial x}+O\left(\beta^{2}\right) .
$$

In the last term in (63) we use the uniform expansion of $F_{n}$ (46) which starts from the third power of $1 / n$. All integrals can be calculated in closed form, their expansion starts from the second order in $\beta$. Therefore

$$
\sum_{n=1}^{\infty} 2 \int_{\beta}^{\infty} d x \sqrt{x^{2}-\beta^{2}} \frac{\partial F_{n}}{\partial x}=\sum_{n=1}^{\infty} 2 \int_{0}^{\infty} d x x \frac{\partial F_{n}}{\partial x}+O\left(\beta^{2}\right)
$$

Puting together all three parts we obtain

$$
D(\beta)=D(0)+\frac{\pi}{2} \xi \beta+O\left(\beta^{2}\right) .
$$

The expansion of the remaining terms in (61) contains the same linear term

$$
\text { const }+\frac{\pi}{2} \xi \beta+O\left(\beta^{2}\right)
$$

which is canceled by that in (64). Therefore the expansion of $G(\beta)$ starts at least from the second power of $\beta$. 

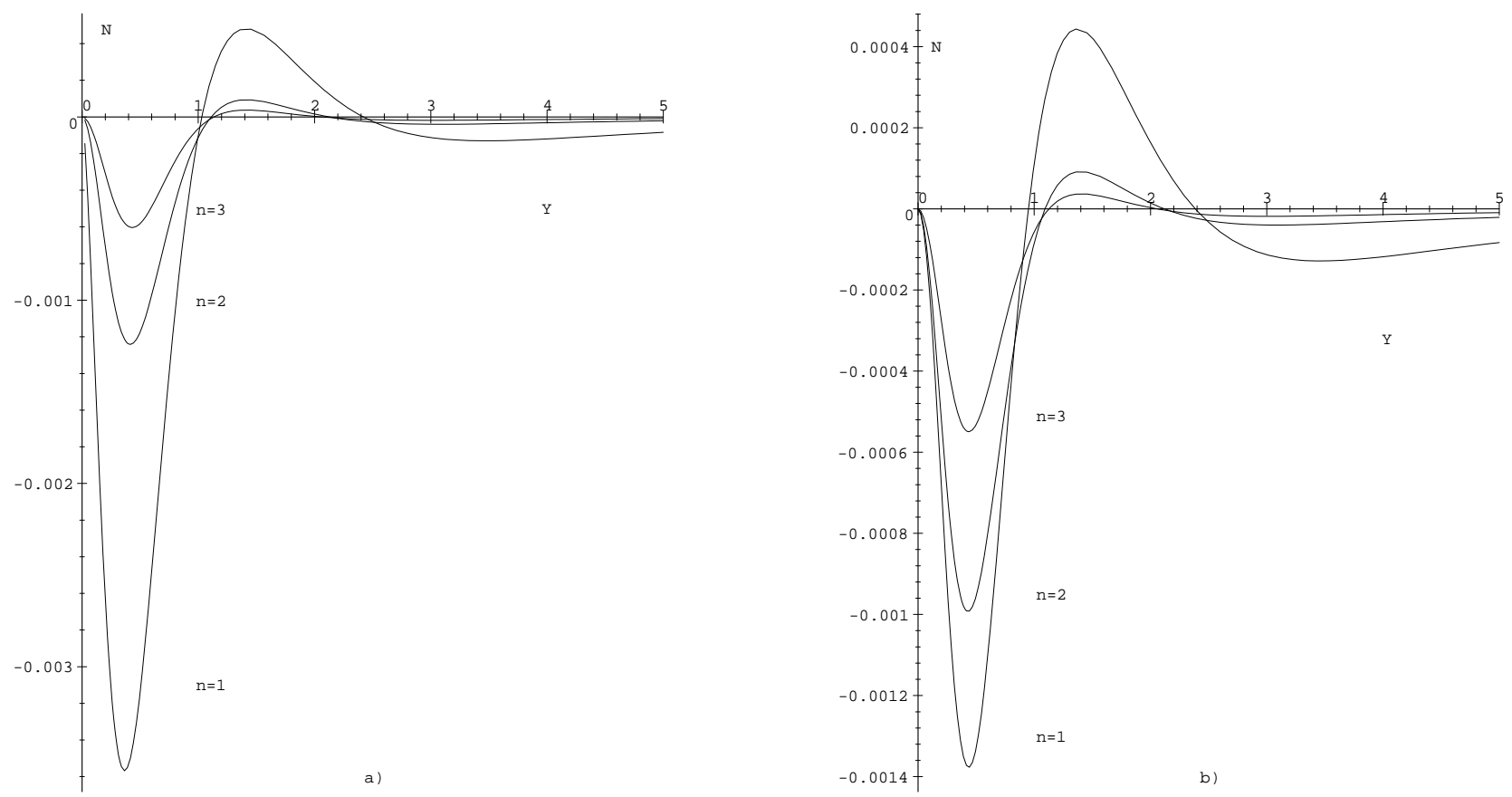

Figure 2: Plots of the integrand $N_{n}=2 n y \frac{\partial}{\partial y} F_{n}\left(\left(n^{2} y^{2}+\beta^{2}\right)^{1 / 2}\right)$ for a) $\beta=0$ and b) $\beta=0.4$ and $n=1,2,3$

For the numerical analysis of the series in $G(\beta)$ first of all we replace the integration variable $x \rightarrow y=\sqrt{x^{2}-\beta^{2}} / n$. Then we obtain

$$
\sum_{n=1}^{\infty} \int_{\beta}^{\infty} 2 \sqrt{x^{2}-\beta^{2}} \frac{\partial}{\partial x} F_{n}(x) d x=\sum_{n=1}^{\infty} \int_{0}^{\infty} 2 n y \frac{\partial}{\partial y} F_{n}\left(\sqrt{n^{2} y^{2}+\beta^{2}}\right) d y .
$$

Some first integrands $N_{n}=2 n y \frac{\partial}{\partial y} F_{n}\left(\sqrt{n^{2} y^{2}+\beta^{2}}\right)$ are plotted in Fig.2 for $\beta=0$ and for $\beta=0.4$. As it is seen from the figures, all functions $N_{n}(y)$ have quite large variations near the origin and decrease as $1 / y^{3}$ for large $y$.

For higher $n,(n>3)$, the Bessel functions entering $F_{n}$, have been substituted by their unifom asymptotic expansions whereby the first 13 terms were taken. The error caused by this approximation is smaller than $10^{-7}$. Than the integral and the sums can be carried out explicitely. For this task Maple was used.

With this, the function $G(\beta)$ was calculated for $0 \leq \beta \leq 2$ and the result

$$
|G(\beta)|<10^{-7}
$$


was obtained numerically.

\section{References}

[1] T.W.B. Kibble, J. Phys. A. 9, (1976) 1387.

[2] A. Vilenkin and E.P.S. Shellard Cosmic strings and other Topological Defects (Cambridge Univ. Press, Cambridge, 1994); M. Hindmarsh and T.W.B. Kibble, Rep. Prog. Phys. 58, (1995) 477.

[3] A. Vilenkin, Phys. Rev. D 23, (1981) 852.

[4] D.D. Sokolov and A.A. Starobinskii, Sov. Phys. Dokl. 22, (1977) 312.

[5] E.M. Serebriany, Theor. Math. Phys. V.64, (1985) 299; T.M. Helliwell and D.A. Konkowski, Phys.Rev. D34, (1986) 1918 ; J.S. Dowker, Phys.Rev. D36, (1987) 3095 ; B. Linet, Phys.Rev. D35, (1987) 536 ; V.P. Frolov, E.M. Serebryany, Phys.Rev. D35, (1987) 3779 ; P.C.W. Davies, V. Sahni, Class. Quant. Grav., V.5, (1988) 1.

[6] B. Linet, Class. Quant. Grav., V.9, (1992) 2429 ; D. Fursaev, Class. Quant. Grav., V.11, (1994) 1431 ; V.P. Frolov, A. Pinzul, and A.I. Zelnikov, Phys.Rev. D51, (1995) 2770 ; G. Cognola, K. Kirsten, and L. Vanzo, Phys.Rev. D49, (1994) 1029.

[7] M. Kac, Am. Math., 73, (1966) 1.

[8] J.R.Gott, The Astrophys. J., 288, (1985) 422.

[9] W.A. Hiscock, Phys. Rev. D31, (1985) 3288.

[10] M. Bordag, E. Elizalde, K. Kirsten, S. Leseduarte, Phys. Rev. D 56, (1997) 4896.

[11] M. Abramowitz and I. Stegun, Handbook of mathematical functions (National bureau of standarts, 1964).

[12] E. Elizalde, S.D. Odintsov, A. Romeo, A.A. Bytsenko and S. Zerbini, Zeta regularization techniques with applications (World Scientific, 1994).

[13] G.Kennedy, R.Critchley and J.S.Dowker, Ann. Phys., 125, (1980) 346.

[14] M.Bordag, J. Phys. A., 28, (1995) 755; M. Bordag, K. Kirsten, Phys. Rev. D 53, (1996) 5753.

[15] M. Bordag, Annalen der Physik, 47, (1990) 93. 
[16] I. Brevik, B. Jensen, Vanishing of Gravitational Particle Production in the Formation of Cosmic Strings, hep-th/9803246. 